\title{
Transverse Ultrasound Assessment of the Displacement of the Median Nerve in the Carpal Tunnel during Wrist and Finger Motion in Healthy Volunteers
}

\author{
Mitsuhiko Nanno ${ }^{1}$, Takuya Sawaizumi ${ }^{2}$, Norie Kodera ${ }^{2}$, \\ Yuji Tomori ${ }^{2}$ and Shinro Takai ${ }^{2}$ \\ ${ }^{1}$ Department of Orthopaedic Surgery, Nippon Medical School Musashi Kosugi Hospital \\ ${ }^{2}$ Department of Orthopaedic Surgery, Nippon Medical School
}

\begin{abstract}
Purpose: The purpose of this study was to investigate the displacement of the median nerve in the carpal tunnel during finger motion at varied wrist positions using transverse ultrasound in healthy volunteers, in order to clarify the appropriate position of a wrist splint in treating carpal tunnel syndrome.

Methods: Fifty wrists of 25 asymptomatic volunteers were evaluated by transverse ultrasound. The location of the median nerve in the carpal tunnel was examined at 5 wrist positions (neutral, $60^{\circ}$ dorsiflexion, $60^{\circ}$ palmar flexion, $40^{\circ}$ ulnar flexion, $10^{\circ}$ radial flexion) with all 5 fingers in full extension, all 5 fingers in full flexion, and isolated thumb in full flexion, respectively.

Results: The median nerve was located significantly $(p<0.05)$ more dorsally at the wrist dorsal flexion position, more ulnopalmarly at the wrist palmar flexion position, more radially at the wrist radial flexion position, and more radially at the wrist ulnar flexion position than at the wrist neutral position in all 5 fingers at full extension. The median nerve moved the most significantly dorsally among all wrist positions during finger motion at the wrist dorsal flexion position $(\mathrm{p}<0.05)$. Conversely, the median nerve moved the most significantly ulnopalmarly at the wrist palmar flexion position with all 5 fingers in full flexion among all wrist positions during finger motion $(\mathrm{p}<0.05)$. This latter wrist and finger position induced significant displacement of the median nerve toward the transverse carpal ligament, and compressed it between the flexor tendons and the transverse carpal ligament.

Conclusions: This study showed that there is a significant relationship between the median nerve displacement in the carpal tunnel and the motion of the wrist and fingers. This finding suggests that the compression or the shearing stress of the median nerve caused by the movement of the flexor tendons is reduced in the wrist dorsal flexion position compared with other wrist positions. This wrist dorsal flexion position could be the appropriate position for a wrist splint in the treatment for carpal tunnel syndrome. This ultrasound information provides further knowledge and understanding of the biomechanics and pathophysiology of the carpal tunnel. It could also help in the accurate analysis and assessment of diagnostic images and treatment for carpal tunnel syndrome.
\end{abstract}

(J Nippon Med Sch 2015; 82: 170-179)

Key words: median nerve, carpal tunnel, ultrasound, wrist position, finger motion

\section{Introduction}

Carpal tunnel syndrome (CTS) is one of the most common entrapment neuropathies of the upper extremity in which the median nerve is compressed within the carpal tunnel. The repetitive motion of the fingers and the wrist has frequently been associated with the development of
$\mathrm{CTS}^{1}$. In particular, a strong pinch or grip associated with wrist flexion is considered to be a risk factor for $\mathrm{CTS}^{2}$. Moreover, repetitive motion may also cause pathological changes in the median nerve and flexor tendons ${ }^{1}$.

Recently, several authors have reported that motion of the fingers and the wrist induces considerable excursion

Correspondence to Mitsuhiko Nanno, Department of Orthopaedic Surgery, Nippon Medical School Musashi Kosugi Hospital, 1396 Kosugi-cho, Nakahara-ku, Kawasaki, Kanagawa 211-8533, Japan

E-mail: nanno-mi@ga2.so-net.ne.jp

Journal Website (http://www.nms.ac.jp/jnms/) 
of the median nerve at the wrist ${ }^{3-8}$. During movements of the fingers or wrist, the median nerve moves longitudinally and transversely in response to the motion of the adjacent tendons that move actively in the carpal tunnel ${ }^{3,7,9}$. Some studies using transverse ultrasonography have demonstrated that the median nerve is displaced palmarly in active finger flexion and becomes compressed between the transverse carpal ligament and the flexor tendons in both healthy persons and patients with $\mathrm{CTS}^{3-7}$. Ultrasonography in vivo could be a useful noninvasive technique to observe and determine mechanical movement quantitatively within the carpal tunnel. Therefore, using ultrasonography to studying the transverse displacement of the median nerve during motions of the wrists and fingers may provide useful information about median nerve kinematic changes in patients with CTS $^{6}$.

Despite the importance of the wrist positions in the pathomechanism of CTS, only a few studies have used ultrasonography to investigate the transverse displacement of the median nerve associated with varied wrist motions $s^{5,6}$. In addition, little research has examined in detail the specific location of the median nerve as a coordinate within the carpal tunnel, although several previous studies have used ultrasonography to evaluated the directions and vectors of the median nerve during finger motion $^{3,4,7,8}$. Demonstrating the location of the median nerve as a coordinate helps provide a more accurate understanding of nerve orientation within the carpal tunnel, such as the start and end points of nerves during wrist and finger motion. Furthermore, the amount of median nerve displacement has previously been examined only as an actual measurement and has not been corrected for individual differences by the size of the carpal tunnel ${ }^{3-8}$.

The purpose of this study was to quantitatively evaluate, with transverse ultrasonography, the location and the displacement of the median nerve as a coordinate within the carpal tunnel at varied wrist positions during finger motion in healthy volunteers. To better understand the kinematics of abnormal nerves in CTS, it is first necessary to identify the movement of normal nerves. This ultrasonographic information could assist in our obtaining a better knowledge and understanding of the biomechanics and pathophysiology of the carpal tunnel. It could also help to improve the analysis and assessment of diagnostic images and the treatment of carpal tunnel syndrome, such as applying a wrist splint.

\section{Materials and Methods}

This study protocol was approved by our institutional re-

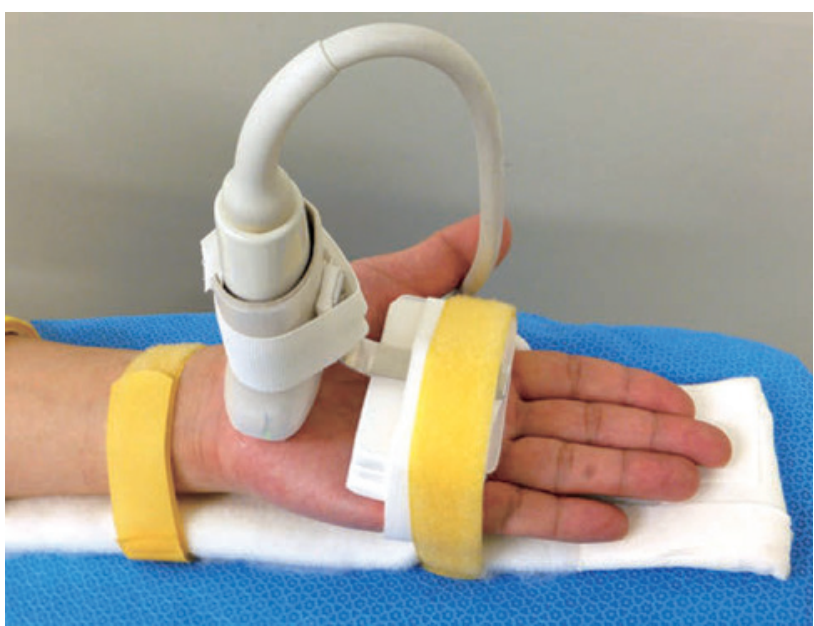

Fig. 1 Ultrasound examination setup at the wrist neutral position

view board. Twenty-five healthy volunteers ( 25 men; age range, 23-56; mean age, 30.9 years) with no history of CTS were recruited. Subjects were excluded if they reported a history of CTS, cervical radiculopathy, peripheral nerve disease, degenerative joint disease, osteoarthritis, rheumatoid arthritis, flexor tendinitis, gout, hemodialysis, amyloidosis, sarcoidosis, thyroid disease, or traumatic injuries to the upper extremities or if they had hand pain or numbness. After written consent was obtained from all subjects, we proceeded with the ultrasonographic examination of both wrists.

\section{Ultrasonographic Examination}

The subjects were imaged sitting with the elbow flexed, the forearm supinated, and the shoulder in neutral position. The forearm was strapped to a custommade table with the wrist in the neutral position. An ultrasonographic scanner (Mylab Five, Hitachi Medical Corporation, Tokyo, Japan) fitted with an LA332 3.5/12 $\mathrm{MHz}$ Hi-Definition linear array transducer (LA332) was used.

Ultrasonographic examination was performed in a standardized manner by a single orthopedic surgeon $(\mathrm{MN})$ trained in the image acquisition procedure. The transducer was positioned at the level of the carpal tunnel with the wrist in the neutral position (Fig. 1). A custom-made transducer-fixing device was fastened at the subject's palm to keep the transducer stable during wrist or finger motion. In addition, the transducer was maintained perpendicular to the skin of the wrist to show both the ridge of the trapezium and the hook of the hamate with reference to a protractor attached to the table, without applying additional pressure to minimize compression of the tissue in the carpal tunnel. These 2 


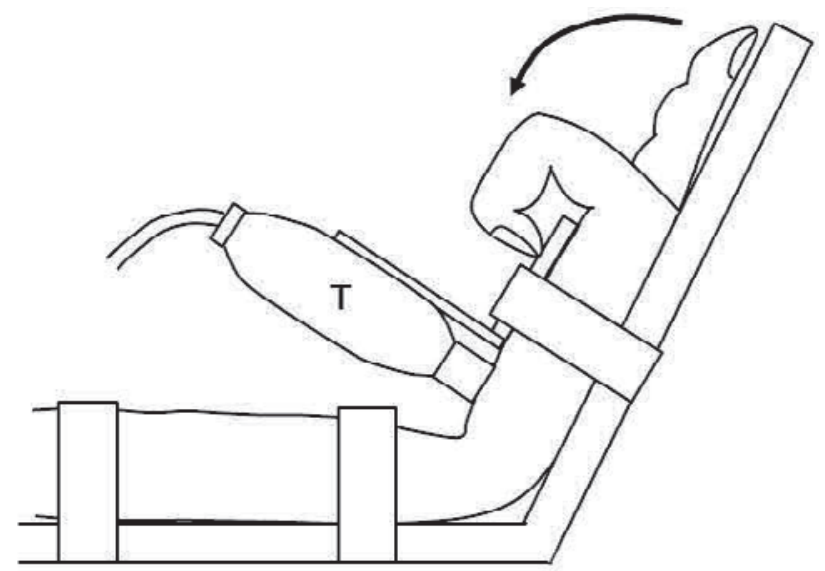

Fig. 2 Illustration of ultrasound examination setup at the wrist palmar flexion position

The transducer $(\mathrm{T})$ was fixed in a custom made fixture during finger motion.

bony landmarks are easily palpable in all hands. The median nerve, the flexor pollicis longus (FPL), the flexor digitorum superficialis (FDS), and profundus tendons (FDP) were identified with transverse ultrasonographic imaging during full flexion and full extension of all 5 fingers and full flexion of the isolated thumb. After a clear image was obtained, the transducer was fixed in a custom-made fixture at each of its 5 study positions (neutral, $60^{\circ}$ dorsiflexion, $60^{\circ}$ palmar flexion, $40^{\circ}$ ulnar flexion, and $10^{\circ}$ radial flexion) during finger motion, respectively (Fig. 2). Each wrist angle was measured and corrected with a goniometer (SD1-01, Suzukiiryoki, Inc., Tokyo) placed on the back of the hand at each of the 5 wrist positions. Both hands of each subject were imaged. The subjects were asked to move from full extension $\left(0^{\circ}\right.$ at each finger joint) to maximum flexion of all 5 fingers as a fist motion, or from full extension of all 5 fingers to maximum flexion of the thumb independently as a single-digit motion at each wrist position. When subjects flexed only the thumb, they actively held the other 4 fingers in extension. Two cycles of the flexion-extension finger motion were recorded for each wrist position.

\section{Image Analysis}

All recorded images were evaluated, and the initial and final frames of the motion for each finger extension and flexion at each wrist position were determined. From these images, the median nerve, FPL, FDS, and FDP were identified in both the extension and flexion positions of the fingers.

The coordinates of the median nerve were determined as follows (Fig. 3). The reference point (P) was defined as the midpoint of the distance $(\mathrm{A})$ between the vertex of

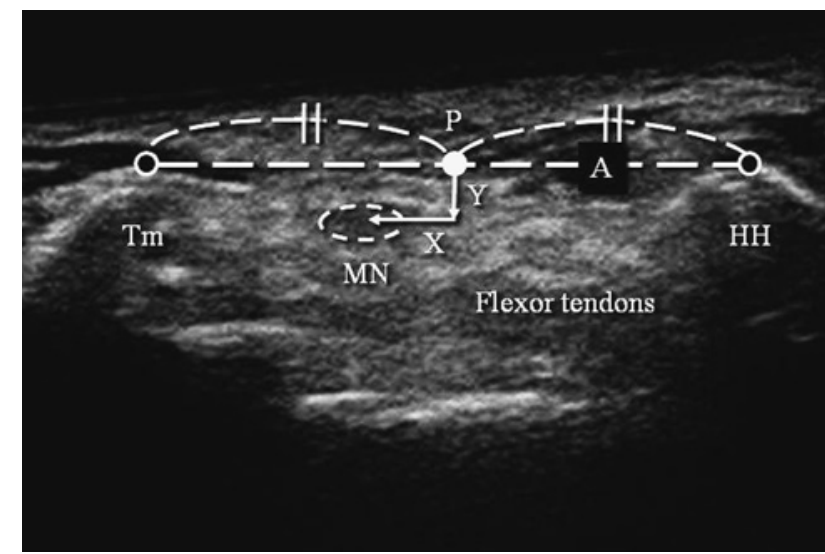

Fig. 3 Measuring the location of the median nerve within the carpal tunnel as a coordinate

$\mathrm{MN}$ : the median nerve, Tm: the trapezium, $\mathrm{HH}$ : the hook of the hamate, Distance ' $\mathrm{A}$ ': the distance between the vertex of the ridge of the Tm and the vertex of the $\mathrm{HH}$, Point ' $\mathrm{P}$ ': the reference point defined as the midpoint of the distance ' $\mathrm{A}$ '

Distance ' $X$ ': The distance between the point ' $\mathrm{P}$ ' and the centroid of $\mathrm{MN}$ on the $\mathrm{X}$-axis

Distance ' $\mathrm{Y}$ ': The distance between the point ' $\mathrm{P}$ ' and the centroid of $\mathrm{MN}$ on the $\mathrm{Y}$-axis

the ridge of the trapezium and the vertex of the hook of the hamate. The distances between the reference point and the centroid of the median nerve on the $X$-axis $(X)$ and on the $\mathrm{Y}$-axis $(\mathrm{Y})$ were measured. The position of the median nerve was shown as a coordinate point (X mm, $Y$ $\mathrm{mm})$. To correct individual differences, the ratio $(x)$ of distance $(X)$ to distance $(A)$ and the ratio $(y)$ of distance $(\mathrm{Y})$ to distance $(\mathrm{A})$ were measured and defined as the radial deviation ratio and the dorsal deviation ratio of the median nerve. The corrected position of the median nerve was shown as a coordinate point $(x, y)$. As the ratio $x$ or the ratio $y$ increased, the location of the median nerve was more deviated to the radial side or the dorsal side. Therefore, the displacement of the nerve in ulnarradial and palmar-dorsal directions could be calculated by comparison with the coordinates at the initial position of the nerve and its final one within the carpal tunnel during finger motion.

\section{Statistical Analysis}

The results were expressed as the mean and standard deviation. The averages of the 3 cycles were calculated. All analyses were performed with the software program IBM SPSS Statistics 21.0J (IBM Japan Ltd., Tokyo, Japan). The displacement of the median nerve from the neutral wrist position to the other 4 wrist positions in all 5 fingers was statistically analyzed with the paired t-test. In addition, the median nerve displacement from extension 


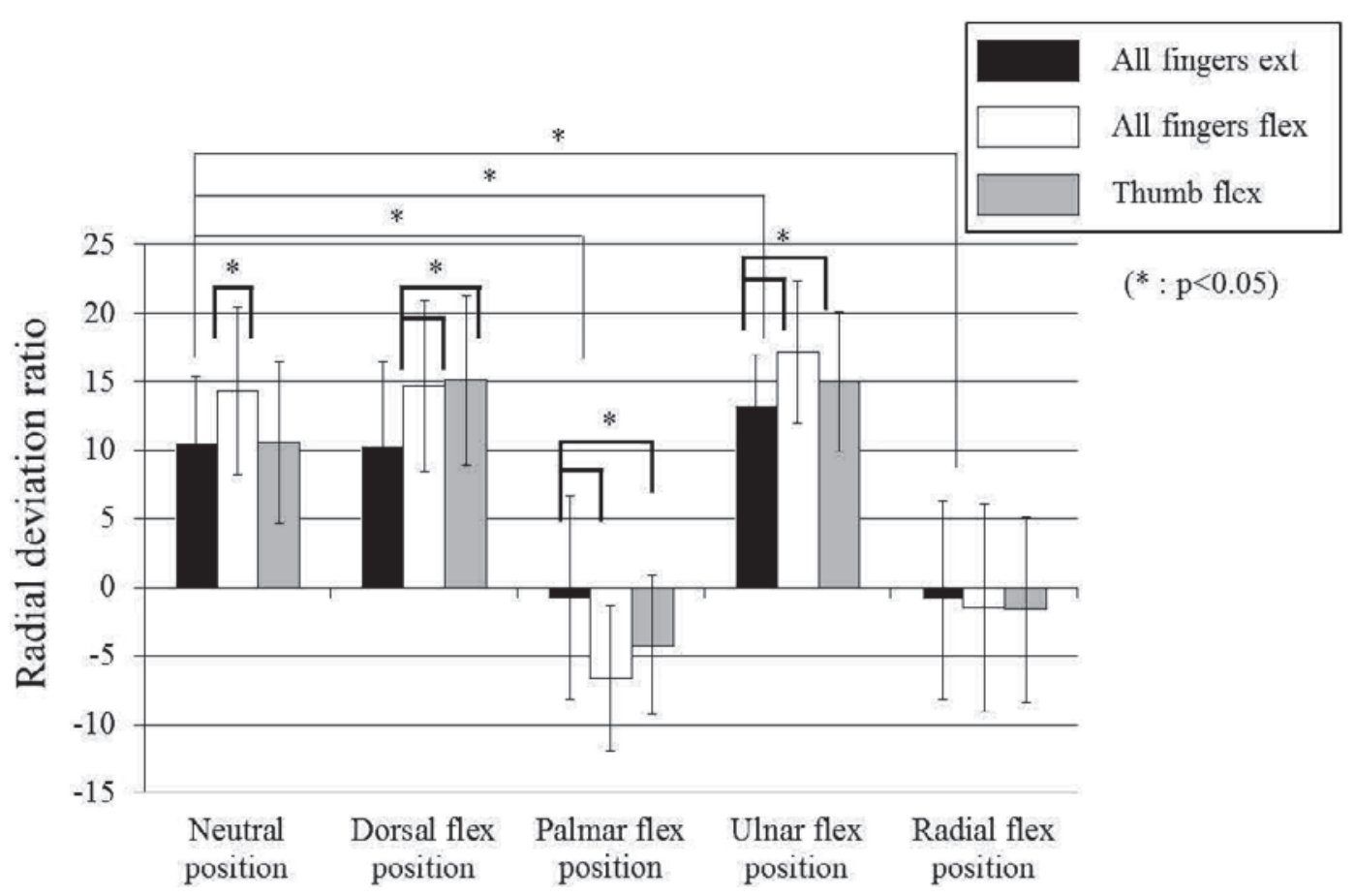

Fig. 4 Position of the median nerve during wrist motion with finger motion (radial-ulnar direction)

to flexion of all 5 fingers and the isolated thumb at each of the 5 wrist positions was statistically analyzed with the paired t-test. A p-value of less than 0.05 was considered to indicate statistical significance for differences in the motion of fingers and wrists.

\section{Results}

1) Location of the Median Nerve in the Radial-ulnar and the Dorsal-palmar Directions during Varied Wrist Motions in the Extension of All 5 Fingers

Locations of the median nerve in the radial-ulnar and the dorsal-palmar directions are shown in Figure 4, 5 . The mean location and standard deviation of the median nerve at the wrist neutral position was $10.5 \pm 4.9$ in the radial-ulnar direction and $11.0 \pm 2.2$ in the dorsal-palmar direction.

In the radial-ulnar direction, the median nerve was located significantly $(p<0.05)$ more ulnarly both at the wrist palmar and radial flexion positions than at the wrist neutral position in all 5 fingers full extension (Fig. 4). The median nerve displacement toward the ulnar side was significantly larger $(\mathrm{p}<0.05)$ at the wrist palmar flexion position than at the radial flexion position. Conversely, the median nerve was located significantly $(p<0.05)$ more radially at the wrist ulnar flexion position than at the wrist neutral position in all 5 fingers full extension. There was no significant difference in the median nerve location between the wrist neutral position and the dorsal position in all 5 fingers full extension.

In the dorsal-palmar direction, the median nerve was located significantly $(\mathrm{p}<0.05)$ more dorsally at the wrist dorsal flexion than it was at the wrist neutral position in the full extension of all 5 fingers (Fig. 5). On the other hand, the median nerve was located significantly $(\mathrm{p}<$ 0.05 ) more palmarly at the wrist palmar flexion position than at the wrist neutral position in all 5 fingers extension. There was no significant difference in the median nerve location among the wrist neutral, ulnar, and radial positions in all 5 fingers full extension.

2) Displacement of the Median Nerve at Varied Wrist Positions during All 5 Fingers Motion in the Radialulnar and the Dorsal-palmar Direction

In the radial-ulnar direction and at the wrist neutral, dorsal, and ulnar flexion positions, the median nerve moved significantly $(p<0.05)$ more radially in the full flexion of all 5 fingers than in full extension (Fig. 4).

At the wrist palmar flexion position, the median nerve moved significantly $(\mathrm{p}<0.05)$ more ulnarly in the full flexion of all 5 fingers than in full extension. At the wrist radial flexion position, the median nerve displacement toward the ulnar side was slightly but not significantly larger in the full flexion of all 5 fingers than in full extension.

In the dorsal-palmar direction, at the wrist dorsal, palmar, radial, and ulnar flexion positions, but not in the neutral position, the median nerve moved significantly 


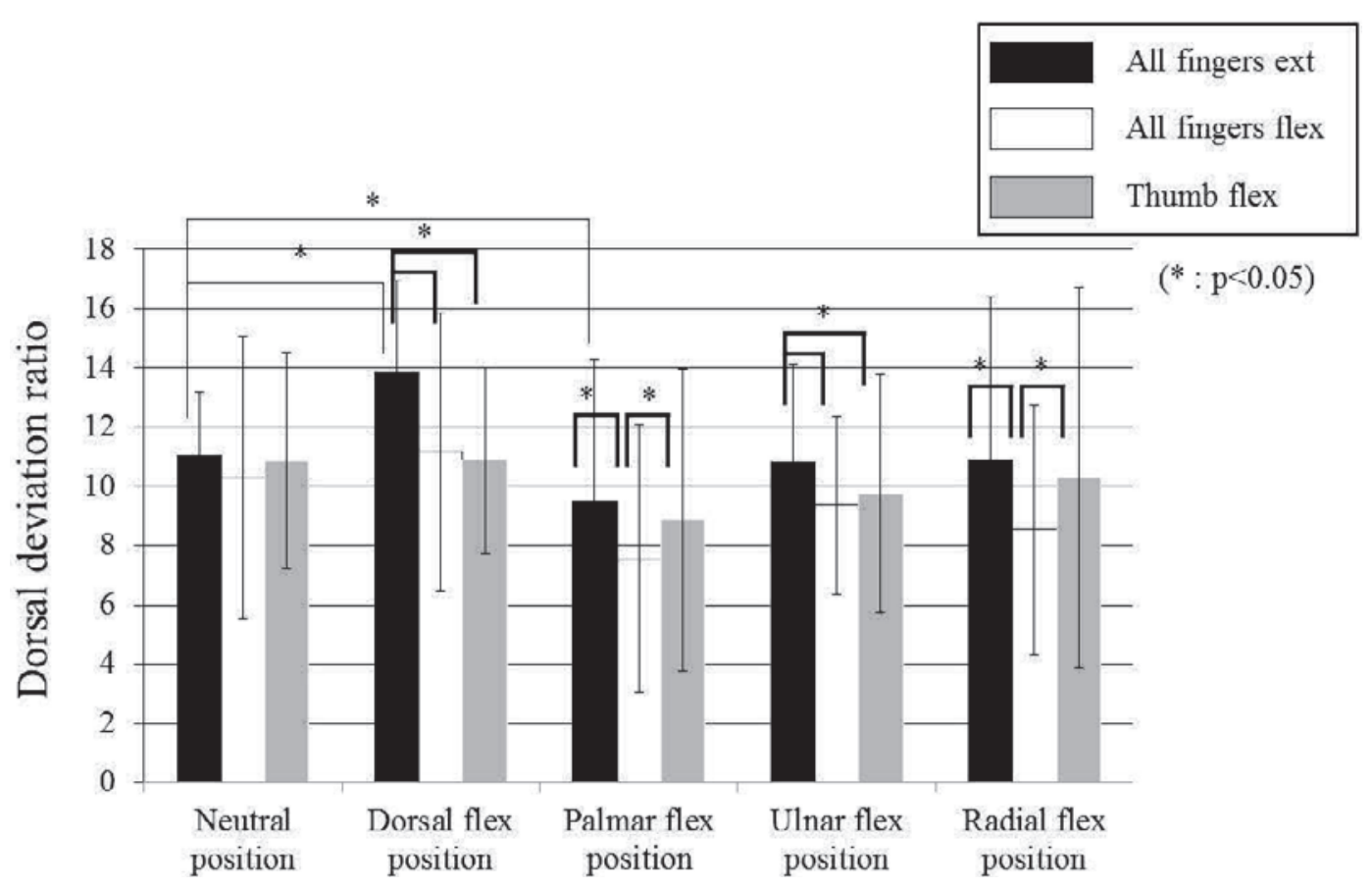

Fig. 5 Position of the median nerve during wrist motion with finger motion (dorsal-palmar direction)

$(p<0.05)$ more palmarly in the full flexion of all 5 fingers than in full extension (Fig. 5). At the wrist neutral flexion position, the median nerve displacement toward the palmar side was slightly but not significantly larger in the full flexion of all 5 fingers than in full extension.

Regarding the flexor tendon motion on the transverse ultrasonographic imaging in all 5 fingers full flexion, the FPL moved radiodorsally, separately from the radiopalmar direction motion of the FDS and FDP at the wrist neutral, dorsal, and ulnar flexion positions. At the wrist palmar and radial flexion positions, the FPL, FDS, and FDP moved ulnopalmarly and pushed the nerve toward the transverse carpal ligament in all 5 fingers full flexion.

3) Displacement of the Median Nerve at Varied Wrist Positions Comparing All 5 Fingers Motion and Isolated

\section{Thumb Motion}

In the radial-ulnar direction, there was no significant difference in the median nerve displacement between all 5 fingers motion and isolated thumb motion (Fig. 4). Furthermore, the median nerve moved the significantly $(\mathrm{p}<$ 0.05) most ulnarly at the wrist palmar flexion position at all wrist positions during finger motion.

In the dorsal-palmar direction, at the wrist palmar and radial flexion positions, the median nerve moved significantly $(p<0.05)$ more palmarly in all 5 fingers flexion than in isolated thumb flexion (Fig. 5). The median nerve displacement toward the dorsal side at the wrist dorsal flexion position and that toward the palmar side at the neutral and ulnar flexion positions were slightly but not significantly larger in all 5 fingers flexion than in isolated thumb flexion. Furthermore, the median nerve moved the most significantly $(\mathrm{p}<0.05)$ palmarly at the wrist palmar flexion position among at all wrist positions during finger motion. In addition, the distance between the median nerve and the transverse carpal ligament in the dorsal-palmar direction was significantly smaller with all fingers motion than with isolated thumb motion $(\mathrm{p}<0.05)$.

\section{Discussion}

CTS is the most common entrapment neuropathy at the wrist and is caused by compression of the median nerve in the carpal tunnel. The major characteristic pathological finding in CTS is thought to be noninflammatory fibrosis and thickening of the subsynovial connective tissue (SSCT), which lies between the flexor tendons and the visceral synovium of the ulnar tenosynovial bursa ${ }^{10,11}$. This fibrosis likely affects the motion characteristics of the SSCT, tendon excursion, and median nerve ${ }^{11}$. The change may also increase the volume of the SSCT and cause elevated shear strain and pressure in the carpal tunnel, which finally can induce $\mathrm{CTS}^{1,12}$. However, repetitive and forceful motion of the fingers and the wrist has been suggested as a risk factor for $\mathrm{CTS}^{13-15}$. In addition, it is generally accepted that this mechanical factor is related to the pathological change of the SSCT typically seen in patients with $\mathrm{CTS}^{1,13}$. 


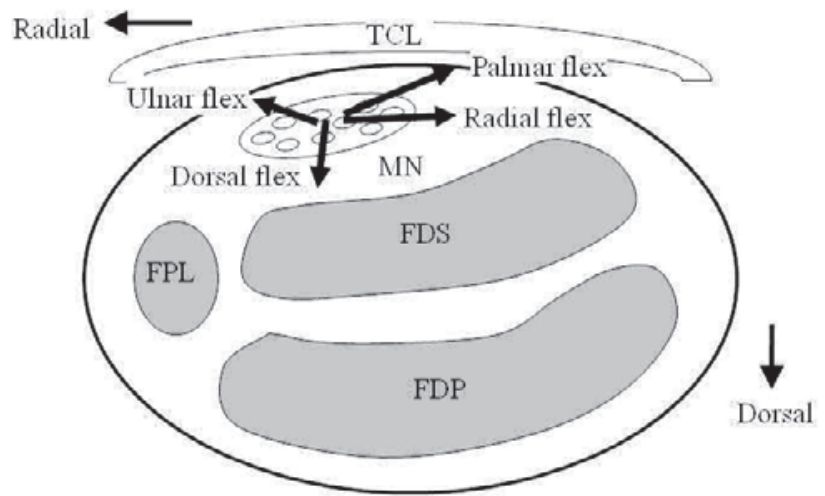

Fig. 6 Illustration of the movement of the median nerve during the wrist motion with the all fingers in extension The nerve position within the carpal tunnel at the wrist neutral position is fixed as a starting point.

$\mathrm{MN}$ : the median nerve, FPL: the flexor pollicis longus, FDS: the flexor digitorum superficialis, FDP: the flexor digitorum profundus, TCL: the transverse carpal ligament

In several recent studies transverse ultrasonography was used to evaluate median nerve motion in the carpal tunnel $^{3-8}$. Studying the transverse movement of the median nerve in the carpal tunnel during finger motion at varied wrist positions is important for understanding the kinematics of the median nerve in both physiologic and pathophysiologic states ${ }^{5,6}$. In particular, ultrasonography is a useful tool for obtaining dynamic information about the median nerve motion throughout the full range of wrist and finger motions in vivo.

Several studies have focused on transverse displacement patterns of the median nerve in the carpal tunnel during finger motion at the wrist neural position, but only a few studies have focused on the median displacement at varied wrist positions ${ }^{5,6}$. A cadaveric study by Yoshii et al found that the shear strain in the SSCT between the median nerve and the tendon is higher at $60^{\circ}$ of wrist flexion than at $30^{\circ}$ of flexion, full wrist extension, or neutral wrist positions ${ }^{16}$. This study also found that the gliding resistance of flexor tendons against the transverse carpal ligament is significantly greater at $60^{\circ}$ of wrist flexion than at the neutral position ${ }^{16}$. A cadaver study by Zhao et al has also shown that wrist position affected tendon gliding resistance, which was highest when the wrist was in $60^{\circ}$ of flexion ${ }^{13}$. Currently, there is little information detailing the transverse displacement of the median nerve within the carpal tunnel compared with various wrist motions by ultrasonography. In an ultrasonographic study in the normal carpal tunnel by Wang et $\mathrm{al}^{6}$, wrist palmar flexion and ulnar flexion were shown to induce significant transverse displacement and deformation of the median nerve. However, this finding is inconsistent with our findings. The difference in results is probably related to the study by Wang et al not evaluating the motion of the median nerve at the radial flexion position ${ }^{6}$ and our study comparing the motion of the median nerve at varied wrist positions, such as the neutral, dorsal flexion, palmar flexion, ulnar flexion, and radial flexion.

Consequently, we found that certain wrist and finger motions resulted in significant displacement of the median nerve in the carpal tunnel on the transverse ultrasonographic imaging in healthy subjects.

In the present study, the median nerve moved significantly $(\mathrm{P}<0.05)$ dorsally at the wrist dorsal flexion position, ulnopalmarly at the wrist palmar flexion position, radially at the wrist ulnar flexion position, and ulnarly at the wrist radial flexion position when the location of the median nerve at the wrist neutral position was defined as a standard (Fig. 6).

The Phalen test ${ }^{17}$ and the Okutsu test ${ }^{18}$, which are the common maneuvers used to provoke symptoms of CTS, are performed at the wrist palmar or radial flexion position without any finger motion respectively. The current study indicates that these positions cause significant transverse displacement of the median nerve in the carpal tunnel. Especially, we found that the median nerve was located most ulnopalmarly in the carpal tunnel at the wrist palmar flexion position with all 5 fingers in flexion.

Moreover, we have found that the transverse displacement of the median nerve within the carpal tunnel is affected not only by the wrist motions, but also by finger motion. We showed that with fist and individual thumb motion, the tendons move towards the median nerve, thereby pushing it in either a radial or ulnar direction and in either a dorsal or palmar direction. Their movements of both the median nerve and the flexor tendons were classified to two patterns according to the wrist position (Fig. 7). In the wrist neutral, dorsal, and ulnar flexion positions, the FDS and the FDP move together palmarly and the FPL separately moves dorsally from all 5 fingers full extension to flexion. As a result, the space in which the FPL had been located becomes vacant after the FPL movement. Then the median nerve moves into that space, that is, in a radiopalmar direction to escape the compression and additionally shifts away from the transverse carpal ligament. Especially, we observed that the median nerve at the wrist dorsal flexion position was lo- 


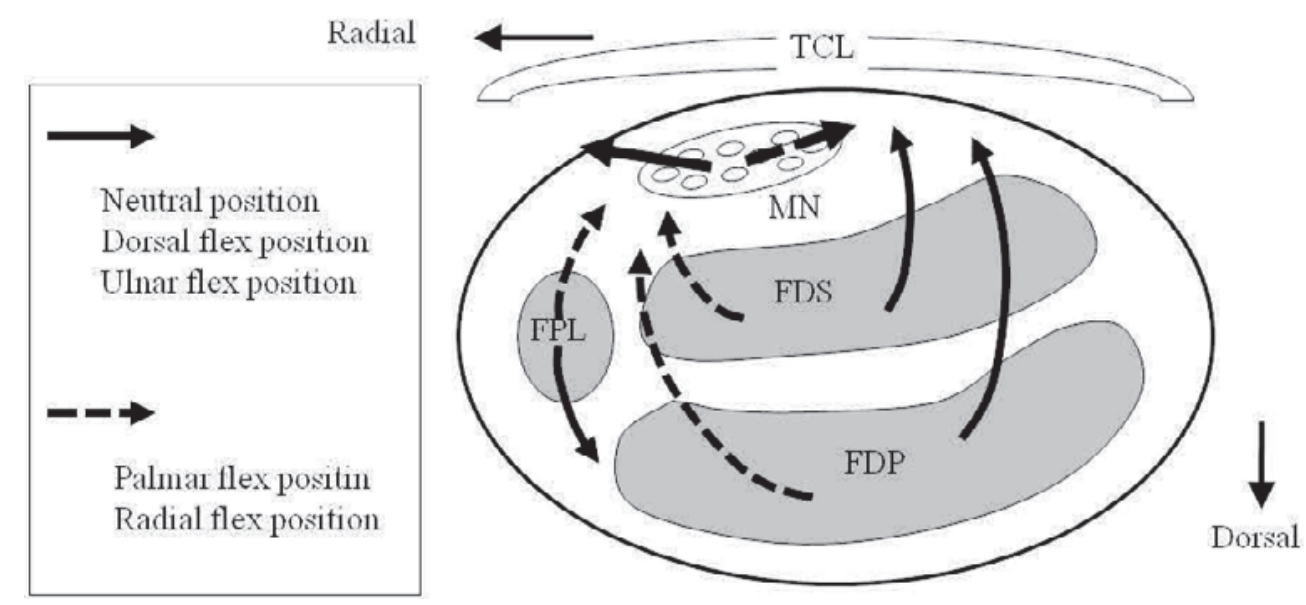

Fig. 7 Illustration of the movement of both the median nerve and the flexor tendons during all 5 fingers flexion at varied wrist flexion positions

MN: the median nerve, FPL: the flexor pollicis longus, FDS: the flexor digitorum superficialis, FDP: the flexor digitorum profundus, TCL: the transverse carpal ligament

cated the most dorsally within the carpal tunnel among at all wrist positions during finger motion. This finding suggests that the compression or the shearing stress of the median nerve caused by the movement of the flexor tendons may be reduced in the wrist dorsal flexion position compared with other wrist positions. Therefore, the wrist dorsal flexion position may be effective in the treatment of CTS with a wrist splint.

On the other hand, at the wrist palmar or radial flexion position, the FDS, the FDP, and the FPL move together ulnopalmarly from full extension to full flexion of all fingers. Consequently, the median nerve is pushed to the ulnopalmar side by the flexor tendons. Finally, it is sandwiched by the flexor tendons and the transverse carpal ligament, and is compressed. As the carpal tunnel is an oval closed space, the median nerve cannot move away from the tendons and thus gets compressed. In this study we have shown that in flexion and extension, regardless of the specific finger motion, the displacement of the median nerve is more ulnopalmarly at the wrist palmar flexion position than at other wrist positions.

There have been few studies on the displacement of the median nerve compared in fist motion and individual thumb motion. Some studies on the nerve displacement during individual thumb, index, or middle finger have been reported. However, the full flexion of the index or middle finger separately is difficult. Therefore, we chose to evaluate fist motion because it is common to many hand activities. We also chose to assess individual thumb motion for two reasons. First, the FPL tendon is separated from others flexor tendons compared to the FDS and the FDP of other fingers. And it is relative easy to acquire ultrasonographic imaging of the FPL. Second, the FPL is neighboring to the median nerve and so may be expected to have more influence on nerve movement than tendons that are more distant from the nerve. We described a significant difference in the median nerve displacement when comparing that in fist and thumb motions. Especially, at the wrist palmar and radial flexion positions, the median nerve moves more ulnopalmarly in full flexion of all 5 fingers than in the individual thumb (Fig. 8).

In the wrist neutral, dorsal, and ulnar flexion positions, the FPL tendon flexes independently, and moves toward the transverse carpal ligament. However, this motion is slightly blocked by the median nerve, and the tendon remains relatively dorsal to the nerve. On the other hand, the FDS and the FDP during fist motion move together ulnopalmarly, so the median nerve slides away from the tendons and moves ulnopalmarly. From these findings, it is speculated that the median nerve may be compressed on the tansverse carpal ligament more strongly in all 5 fingers flexion than in individual FPL flexion at the wrist palmar and radial flexion positions.

Although a number of previous studies on the displacement of the median nerve by transverse ultrasound have been reported, many authors positioned the transducer on the wrist crease, which was thought to be the proximal level of the carpal tunnel. There is a possibility that its position was varied for each subject or each examination even for the same subject, because the landmark for examination was the mobile skin. In the current study, the transducer was fixed at the distal level of the carpal tunnel to show both the ridge of the trapezium 


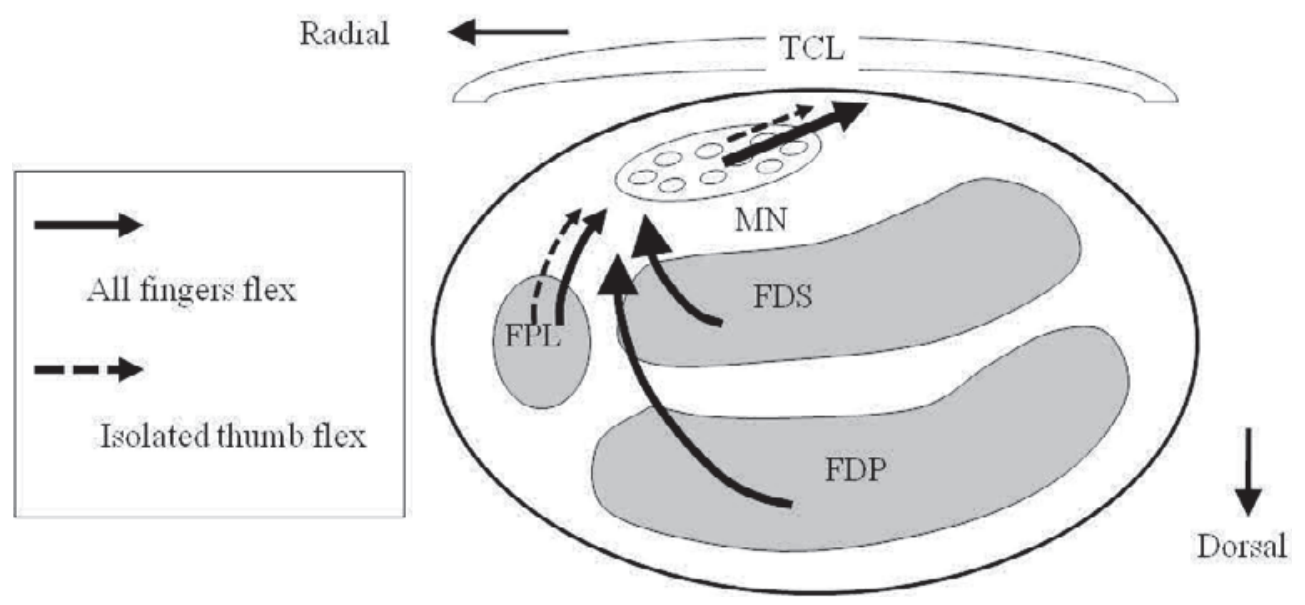

Fig. 8 Illustration of the movement of both the median nerve and the flexor tendons during all 5 fingers flexion and isolated thumb flexion at the wrist palmar or radial flexion position MN: the median nerve, FPL: the flexor pollicis longus, FDS: the flexor digitorum superficialis, FDP: the flexor digitorum profundus, TCL: the transverse carpal ligament

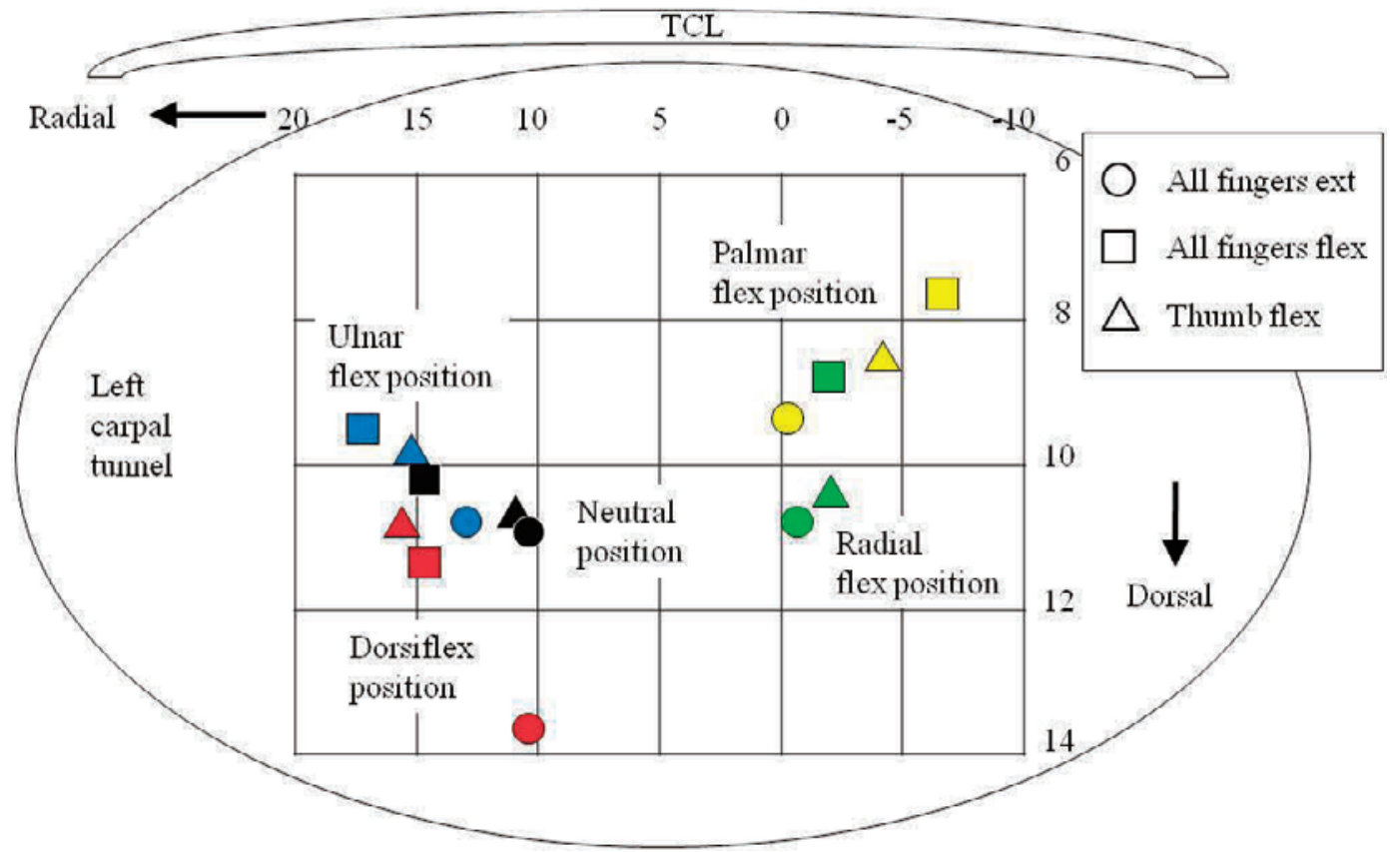

Fig. 9 Location of the median nerve during wrist and finger motion within the carpal tunnel (Average value)

TCL: the transverse carpal ligament

and the hook of the hamate. We used two bony landmarks for the examination to reduce the measurement error for each examination. These landmarks were easily palpable in all hands. The image at the distal carpal tunnel may also be useful as this is a common site at which to examine the median nerve compression clinically.

Moreover, there has been little information demonstrating the specific location of the median nerve as a coordinate within the carpal tunnel, although some previous reports evaluated the directions and the vectors of the me- dian nerve during finger and wrist motions by ultrasound. Detailing the location of the median nerve as a coordinate can offer further accurate understanding of the nerve orientation within the carpal tunnel, that is, the start and end points of the nerve during wrist and finger motions. Furthermore, several authors have evaluated the amount of the median nerve displacement as an actual measurement, and have not corrected for individual differences by the size of the carpal tunnel ${ }^{3-8}$. All data evaluated in this study were corrected by the distance between 
the vertex of the ridge of the trapezium and the vertex of the hook of the hamate.

In the current study, the accurate location and the displacement of the median nerve as a coordinate within the carpal tunnel were evaluated quantitatively at varied wrist positions during finger motions using transverse ultrasound in healthy volunteers. These findings are summarized in Figure 9.

This study showed that there is a significant relationship between median nerve displacement in the carpal tunnel and wrist and finger motions.

This finding suggests that the compression or the shearing stress of the median nerve in response to the flexor tendons' motion would be reduced in the wrist dorsal flexion position compared with others wrist positions. In addition, this wrist dorsal flexion position could be the appropriate position for a wrist splint in the treatment for CTS. Furthermore, we do believe that the examination of the median nerve displacement in the ulnoplamar direction would be a useful next step towards using ultrasound to detect CTS in the future. As we have found that the median nerve is located most ulnopalmarly in wrist palmar flexion, this information may be helpful to understand the pathology of CTS.

This ultrasound information can offer further knowledge and understanding of the biomechanics and pathophysiology of CTS. It could also help in the accurate analysis and assessment of diagnostic images and treatment of CTS when applying such as a wrist splint.

There are some limitations to this study. First, our study has a small sample size. A large sample size would better clarify variations and improve the accuracy in the displacement of the median nerve. Second, we did not evaluate intra- and interobserver differences. Ultrasound measurements are known to be greatly examiner- and experience-dependent, especially with regard to transducer placement. Since all measurements were performed by the same examiner, we were not able to describe interobserver reliability. Additionally, in this study, a custom-made transducer fixing device was fastened at the patient's palm and a custom-made table to keep the transducer stable during wrist or finger motion. Once the transducer was held in position, the examiner could focus only on the screen during finger motion, expectantly reducing examiner dependency. Finally, the kinematics of the median nerve was identified only in normal subjects. However, we believe that this is a necessary first step before investigating abnormal conditions. Accordingly, the data shown in the current study may be useful as base- line data for future studies in patients with CTS. Now that we have this normal data, we plan to perform a similar study to compare it to measurements in patients with CTS in the future.

Conflict of Interest: The authors declare no conflict of interest.

\section{References}

1. Ugbolue UC, Hsu WH, Goitz RJ, Li ZM: Tendon and nerve displacement at the wrist during finger movements. Clin Biomech 2005; 20: 50-56.

2. Feuerstein M, Fitzgerald TE: Biomechanical factors affecting upper extremity cumulative trauma disorders in sign language interpreters. J Occup Med 1992; 34: 257-264.

3. van Doesburg MHM, Yoshii Y, Villarraga HR, Henderson J, Cha SS, An KN, Amadio PC: Median nerve deformation and displacement in the carpal tunnel during Index finger and thumb motion. J Orthop Res 2010; 28: 13871390.

4. van Doesburg MHM, Henderson J, Mink van der Molen $\mathrm{AB}, \mathrm{An} \mathrm{KN}$, Amadio PC: Transverse plane tendon and median nerve motion in the carpal tunnel: ultrasound comparison of carpal tunnel syndrome patients and healthy volunteers. PLoS One 2012; 7: e37081.

5. Wang Y, Zhao C, Passe SM, Filius A, Thoreson AR, An KN, Amadio PC: Transverse ultrasound assessment of median nerve deformation and displacement in the human carpal tunnel during wrist movements. Ultrasound in Med \& Biol 2014; 40: 53-61.

6. Wang Y, Filius A, Zhao C, Passe SM, Thoreson AR, An $\mathrm{KN}$, Amadio PC: Altered median nerve deformation and transverse displacement during wrist movement in patients with carpal tunnel syndrome. Acad Ragiol 2014; 21: 472-480.

7. Yoshii Y, Villarraga HR, Henderson J, Zhao C, An KN, Amadio PC: Ultrasound assessment of the displacement and deformation of the median nerve in the human carpal tunnel with active finger motion. J Bone Joint Surg Am 2009; 91: 2922-2930.

8. Yoshii Y, Ishii T, Tung WL, Sakai S, Amadio PC: Median nerve deformation and displacement in the carpal tunnel during finger motion. J Orthop Res 2013; 31: 1876-1880.

9. Erel E, Dilley A, Greening J, Morris V, Cohen B, Lynn B: Longitudinal sliding of the median nerve in patients with carpal tunnel syndrome. J Hand Surg Br 2003; 28: 439443.

10. Ettema AM, Amadio PC, Zhao C, Wold LE, An KN: A histological and immunohistochemical study of the subsynovial connective tissue in idiopathic carpal tunnel syndrome. J Bone Joint Surg Am 2004; 86: 1458-1466.

11. Ettema AM, Zhao C, Amadio PC, O'Byrne MM, An KN: Gliding characteristics of flexor tendon and tenosynovium in carpal tunnel syndrome: A pilot study. Clin Anat 2007; 20: 292-299.

12. Lluch AL: Thickening of the synovium of the digital flexor tendons: cause or consequence of the carpal tunnel syndrome? J Hand Surg Br 1992; 17: 209-212.

13. Zhao C, Ettema AM, Osamura N, Berglund LJ, An KN, Amadio PC: Gliding characteristics between flexor tendons and surrounding tissues in the carpal tunnel: a biomechanical cadaver study. J Orthop Res 2007; 25: 185190. 
14. Latko WA, Armstrong TJ, Franzblau A, Ulin SS, Werner RA, Albers JW: Crosssectional study of the relationship between repetitive work and the prevalence of upper limb musculoskeletal disorders. Am J Ind Med 1999; 36: 248-259.

15. Szabo RM: Carpal tunnel syndrome as a repetitive motion disorder. Clin Orthop 1998; 351: 78-89.

16. Yoshii Y, Zhao C, Zhao KD, Zobitz ME, An KN, Amadio PC: The effect of wrist position on the rerative motion of tendon, nerve, and subsynovial connective tissue within the carpal tunnel in a human cadaver model. J Orthop Res 2008; 26: 1153-1158.
17. Tetro AM, Evanoff BA, Hollstien SB, Gelberman RH: A new provocative test for carpal tunnel syndrome: Assessment of wrist flexion and nerve compression. J Bone Joint Surg Br 1998; 80: 493-498.

18. Yoshida A, Okutsu I, Hamanaka I: A new diagnostic provocation test for caral tunnel syndrome: Okutsu test. Hand Surg 2010; 15: 65-69.

(Received, November 10, 2014)

(Accepted, February 27, 2015) 